University of Nebraska - Lincoln

DigitalCommons@University of Nebraska - Lincoln

Faculty Publications, Department of Child, Youth, and Family Studies

Child, Youth, and Family Studies, Department of

December 2007

\title{
Marital Satisfaction and Depression: A Replication of the Marital Discord Model in a Latino Sample
}

\author{
Cody S. Hollist \\ University of Nebraska Lincoln, chollist2@unl.edu \\ Richard Miller \\ Brigham Young University \\ Olga G. Falceto \\ Universidade Federal do Rio Grande do Sul, Brazil \\ Carmen Luiza C. Fernandes \\ Community Health Service, Grupo Hospitalar Conceição, Brazil
}

Follow this and additional works at: https://digitalcommons.unl.edu/famconfacpub

Part of the Family, Life Course, and Society Commons

Hollist, Cody S.; Miller, Richard; Falceto, Olga G.; and Fernandes, Carmen Luiza C. , "Marital Satisfaction and Depression: A Replication of the Marital Discord Model in a Latino Sample" (2007). Faculty Publications, Department of Child, Youth, and Family Studies. 48.

https://digitalcommons.unl.edu/famconfacpub/48

This Article is brought to you for free and open access by the Child, Youth, and Family Studies, Department of at DigitalCommons@University of Nebraska - Lincoln. It has been accepted for inclusion in Faculty Publications, Department of Child, Youth, and Family Studies by an authorized administrator of DigitalCommons@University of Nebraska - Lincoln. 
This article is based on the dissertation of Cody S. Hollist. Correspondence concerning this article should be addressed to Cody S. Hollist, Ph.D., University of Nebraska-Lincoln, 130 Mabel Lee Hall, Lincoln, NE 68588-0236. Tel 402 472-8105; fax 402 472-9170; email: cody_hollist@unl.edu

\title{
Marital Satisfaction and Depression: A Replication of the Marital Discord Model in a Latino Sample
}

\author{
Cody S. Hollist, Ph.D. ${ }^{\dagger}$ \\ Richard B. Miller, Ph.D. ${ }^{\ddagger}$ \\ Olga G. Falceto, M.D., Ph.D.§ \\ Carmen Luiza C. Fernandes, M.D.†† \\ † Assistant Professor, Child, Youth, and Family Studies, University of Nebraska-Lincoln \\ $\ddagger$ Professor, School of Family Life, Brigham Young University \\ $\S$ Director of Child and Adolescent Psychiatry, Medical School, \\ Universidade Federal do Rio Grande do Sul, Brazil \\ †† Community Health Service, Grupo Hospitalar Conceição, Brazil
}

\begin{abstract}
The Marital Discord Model of Depression maintains that marital discord is an important antecedent in the development of depression. Although empirical evidence supports this premise, none of this research has been done with Latinos. The purpose of this study was to test the longitudinal relationship between marital satisfaction and depression among 99 Brazilian women. Using structural equation modeling, results indicated that marital satisfaction was a strong predictor of depression 2 years later. Marital satisfaction was also related to cooccurring depression. These results provide evidence that the Marital Discord Model of Depression is an appropriate theoretical model for the conceptualization of marital discord and depression with Latina women and suggest the potential utility of using couples therapy for treating depression among this population.
\end{abstract}

Keywords: Depression, Marital Satisfaction, Marital Discord Model of Depression, Latino

$\mathrm{T}$ he Marital Discord Model of Depression theorizes that marital discord is an important risk factor for depression among many married people (Beach, Sandeen, \& O'Leary, 1990). Although they recognize that the etiology of depression is often multifaceted, these authors argue that the decreased social support and increased hostility in troubled relationships can precipitate depressive symptomotology. Previous research (Beach, Katz, Kim, \& Brody, 2003; Dehle \& Weiss, 1998; Whisman \& Bruce, 1999) has generally found empirical support 
for the model. However, a major weakness of these studies is that they have not used significant samples of minorities, especially Latinos. It is important to understand the relationship between marital discord and depression among Latinos.

\section{Marital Dissatisfaction and Depression Research}

The relationship between marital dissatisfaction and depression is supported by ample evidence that links these variables both cross-sectionally and longitudinally. A meta-analysis of 26 cross-sectional research studies relating marital quality to depression found an effect size of -.42 for women and -.37 for men (Whisman, 2001). In addition, longitudinal research has found that marital distress is a significant predictor of subsequent depression (Dehle \& Weiss, 1998; Schafer, Wickrama, \& Keith, 1998; Whisman \& Bruce, 1999). A seminal article published by Beach et al. (2003) related marital satisfaction and depression using a structural model that linked Time 1 marital satisfaction to Time 1 and Time 2 depression. They found that not only was there an association between marital satisfaction at Time 1 and depression at Time 2, but there was also a cross-lag association between marital satisfaction at Time 1 and spouse's depression at Time 2.

Although longitudinal studies have enhanced our understanding of the relationship between marital quality and subsequent depression, the research has been characterized by a lack of ethnic diversity in the samples. Only one longitudinal study reported the inclusion of Latinos, with only $1 \%$ being Latino (Whisman \& Bruce, 1999). Whisman (2001) has challenged researchers to engage in what he called the "next generation" of research, which focuses on the factors that influence the level of association between marital satisfaction and depression. He urged researchers to study ethnicity in relation to marital quality and depression, explaining that there may be culturally based moderating factors that increase or decrease the relationship between variables. In addition, it is important to test the applicability of Beach's model (Beach, et al., 1990) on a Latino population. The purpose of this study is to test this model by exploring the relationship between marital satisfaction and depression among Latina women in Brazil using longitudinal data.

\section{Characteristics of Marriage and Depression in Brazil}

Epidemiological research indicates that about $10 \%$ of Latino adults living in South American countries in general (Parada, Stevenson, Saldivia, Lohn, \& Torres, 2002), and Brazil specifically (Almeida-Filho et al., 1997), qualify for a diagnosis of major depression. Studies regarding depression in Brazil characterize the symptoms and etiology very similarly to the diagnostic criteria used in the United States. Evidence of this can be seen in the Portuguese translation and validation of the Beck Depression Inventory (BDI; Beck, Rush, Shaw, \& Emery, 1982). It was determined that the depression symptoms used in the BDI were similar to depression symptoms reported by clinicians in Brazil, and thus, the items were merely translated (Beck et al.).

Although the characteristics of depression are similar cross-culturally, characteristics of Latino marriages are different. Latino marriages are largely hierarchical (Korin, 1996), in which roles and responsibilities are clearly defined, with women traditionally responsible for family nurturance. Most Brazilian women continue to regard themselves as primarily responsible 
for caretaking in the family (Korin). These role differences may have an impact on the degree to which marital satisfaction is related to depression, especially for the Latina, who traditionally feel more responsible for the marital relationship than do the men. This perception of increased responsibility on the part of the women may contribute to an increase in the association for women and a decrease for men.

For a majority of Brazil, socioeconomic level has a large impact on marriage. Because the cost of obtaining a marriage license is almost equivalent to the monthly minimum wage, many poor and middle-class individuals in Brazil do not get legally married. Many low-income individuals perform informal ceremonies to celebrate their union. With mean income and education levels lower in Brazil than the United States, many of the individuals who have been studied have marriages arranged by common law. However, in the individual's mind, and in terms of research, it is generally accepted that individuals who consider themselves married are categorized as married. They continue to value the marriage relationship and remain committed to their spouses.

The population of Brazil is racially diverse. Both Europeans and Africans immigrated to South America. Thus, the Brazilian population includes Whites of European descent, Blacks of African descent, and people who are native to the region, combined with interracial marriages. However, the culturally distinctive aspects of these races have largely blurred over the past century, creating a culture that speaks the common language of Portuguese and adheres to Latino cultural norms that are consistent with other Latin and South American countries.

This longitudinal study will focus on two primary hypotheses. First, marital satisfaction will be related to cooccurring depression (i.e., marital satisfaction at Time 1 and depression at Time 1) among Latina women, controlling for age, race, income, and education. Second, marital satisfaction at Time 1 will predict depression at Time 2 among the Latina Brazilian sample of women, after controlling for initial depressive symptomatology, age, race, income, and education. Based on the literature about Brazilian relationships, we hypothesize that the relationship between marital satisfaction and depression will be stronger for this population than for Caucasians.

\section{METHODS}

\section{Procedures}

The data for this study were part of a larger study of Brazilian family development. Data were collected in collaboration with a Brazilian university medical school, the hospital organization Grupo Hospitalar Conceição, and the Family Therapy Institute in Porto Alegre, Brazil. Porto Alegre uses a system of socialized medicine, which is divided into geographic regions that are serviced by hospitals, clinics, and ancillary services. Within each region, there are many districts with a health clinic in each district. These health clinics provide primary care services, like those provided by general practitioners, and are responsible for the medical services of a certain geographic area comprising about 6,000 people. The hospital group responsible for the area in which the study was conducted was Grupo Hospitalar Conceição. It is the second largest medical provision group in Brazil. The study was conducted in conjunction with three medical clinics, where about 18,000 people live. 
The birth of children is registered by the local government, and the information is sent to the health system where the family lives. With this notification, the researchers were informed of potential participants. All children born between March 1999 and May 2000 who lived in the areas covered by the three clinics were considered eligible for the study. When the infants reached 4 months of age, a medical student visited each household to invite them to participate in the study, obtain informed consent, and collect demographic data about the family.

\section{Data Collection}

Interviews were performed by two psychiatrists and/or family therapists. Therapist companionships were interchanged, allowing therapists to conduct interviews with a number of different companions to prevent evaluation biases. The interviews were semistructured and lasted about 2 hours. During this time, therapists observed the behaviors and interactions of the family members and collected the questionnaire data.

The second wave of data collection was conducted when the children were 2 years old. Trained therapists again conducted the interviews. Because of a lack of resources available to conduct the study, data were only collected from the mother and child during the second wave; no fathers' data were collected.

During the initial data collection period, there were 228 infants born. Seven families refused to talk to the interviewer. All other families agreed to participate, signed informed consent, and provided demographic information. Seventy-five families did not finish the initial study because of lack of time availability (mainly concerning the father). After three followup attempts were made, they were considered lost from the sample. Complete data were collected on $67 \%$ of the eligible families contacted.

Because $97 \%$ of individuals reported their demographic data, regardless of whether they completed the study, $t$ tests were run to test the demographic similarity of the remaining participants to those who did not complete the study. Based on these tests, no evidence of sample selection bias was found.

Of the 153 participants who completed data collection for the first wave, 125 participated in the second wave of data collection, which represents an $18 \%$ loss from Time 1 to Time 2. Losses were mainly due to family relocation. To determine if there were any demographic differences between those individuals who completed the second wave of the study and those who were lost, $t$ tests were again run, but no significant differences were found between the two groups. Thus, there was no evidence of attrition bias in the longitudinal sample (Miller \& Wright, 1995).

\section{Sample Characteristics}

Because only mothers responded to both waves of the study, fathers were excluded from these analyses. Twenty-six women were excluded from the analysis because of lack of a partner, leaving 99 women in the final data analysis. The mothers' mean age at Time 1 was 25.9 years old, with range of 14 to 45 . There was a wide range in the level of education among the women. The mean number of years of education was 7.0, with a range of 1 to 14 , indicating that most of the women had little more than an elementary school level of education. The mean number of children for Time 1 was 2.2 , and it was 2.4 at Time 2 . Only $15 \%$ of the fam- 
ilies had additional children born between the first and second waves. The mean number of people living in the home was 4.9 .

Although the population is considered Latina by virtue of country of residence, $58 \%$ were White, 27\% were Black, and 14\% reported a mixed racial heritage. Southern Brazil has a strong European ethnic influence, which accounts for the larger proportion of White participants than may be expected from other regions of Brazil.

The sample was economically representative of this area of Brazil, with the majority being lower middle-class families. Seventy-three percent of the fathers were working at Time 1, and $20 \%$ of the mothers were working outside the home. At the time of the second wave of data collection, the mean family income had dropped. All households had running water and electricity. This sample was similar demographically to many other Brazilian urban populations.

\section{Measurement}

\section{Depression}

Depression was measured in two ways. The first measure was the SRQ-20 (Harding et al., 1980), which was developed in collaboration with the World Health Organization as a triage mental health evaluation in developing countries. The SRQ-20 has been used in many triage situations and studies in Brazil and other countries. It was created through a series of evaluations of 1,624 patients in four countries and demonstrated good reliability and validity (Harding et al.). Scores for the SRQ-20 can range from 0 to 20, with 0 representing absence of depressive symptomatology and 20 the maximum presence of symptomotology.

The instrument was created in multiple languages; therefore, translation and validation procedures were conducted simultaneously with instrument development. The instrument was validated in Brazil, with correlation between clinical assessment and instrument at .70 (Mari $\&$ William, 1986). They reported that the SRQ-20 was an accurate and appropriate screening measure of mental health symptomatology in Brazil.

Because the instrument was not designed to merely assess depression, four tests were used to validate its utility for depression screening in this study. The first was a face validity evaluation. The items in the instrument were checked against the criteria of depression provided in the American Psychiatric Association (2000) and found to be appropriate. The second method of validation was to compare the scores with scores from the BDI. During the second wave of data collection, the Portuguese BDI was used. Although this instrument could not be used in these analyses because it was not available at Time 1 , the Time 2 scores were used to test equivalence between the SRQ and BDI. A correlation of .71 $(p<.001)$ indicated high association between the two instruments. The third method of validation was conducted through a correlation between the SRQ and the therapist's assessment from the interview. This yielded a correlation of $.60(p<.001)$ for Time 1 and $.64(p<.001)$ for Time 2, further indicating the utility in evaluation of depression symptomatology. Finally, another Brazilian study concluded that the SRQ-20 was particularly effective at evaluating depression (Gorenstein, Andrade, Filho, Tung, \& Artes, 1999). Based on these results, the SRQ-20 was considered to be a valid measure of depression symptomatology.

In conjunction with the SRQ-20, therapists provided evaluations of the participants. Trained therapists assessed the degree to which, if any, depression impacted the participants' lives. Throughout the course of the interview, clinicians gathered data and asked questions to better 
understand the mental status of the participants and then used this information to orient their clinical ratings. Scores on this evaluation ranged from 1, indicating no presence of disturbance related to depression, to 3, indicating severe disturbance. Interrater agreement was established by having the participants interviewed and evaluated by two trained therapists who later discussed their assessment and arrived at a diagnostic consensus. The consensus score was used in the evaluation.

This assessment was strongly associated with the SRQ-20 scores. In addition, similar to the validation of the SRQ-20, the Time 2 therapist ratings were correlated with Time 2 BDI scores to verify concurrent validity. The correlation between therapist rating scores and the BDI was $.59(p<.001)$. These psychometric tests indicate that the therapists' evaluations are a valid measure of depressive symptomotology. Although the same therapists administered the paperpencil assessment of depression and did the clinical evaluation, they did not score the written assessment until after they had assigned the clinical score, thus preventing bias from the SRQ20 results on their clinical evaluation.

\section{Marital satisfaction}

There are currently no instruments translated and validated to assess marital satisfaction in Brazil, so a psychometrically established measure could not be used in these analyses. There were four items on the Time 1 questionnaire that were designed to assess respondents' perceptions of marital satisfaction. To establish the credibility of the measure, a confirmatory factor analysis (CFA) was run on the latent variable marital satisfaction. SEM was used to compute the structural model and estimate the factor loadings. Three of the four items were significantly related to the latent variable of marital satisfaction, with the item not related asking about the frequency of arguments in the relationship. Other research has likewise suggested that frequency of arguments is not a distinguishing characteristic of marital satisfaction (Gottman, 1999). Based on these results, this item was removed from the assessment measure. The final CFA model had excellent goodness of fit measures and resulted in standardized estimates of .741 (for global satisfaction), .523 (sexual satisfaction), and .532 (satisfaction with communication). In addition, bivariate correlations of the three variables were all significantly correlated with each other. Taken together, these results suggest that the three measures are adequate measures of the latent variable, marital satisfaction.

Three remaining items were used to assess perceptions of marital satisfaction. The first question was (translated into English), "Are you content with the way that your companion communicates with you what he feels and thinks"? This question was rated on a 3-point Likert scale, with 1 being content and 3 being not content. The second question was, "How is your relationship with your companion"? A 3-point Likert scale ranged from 1 (good) to 3 (very difficult). The third question was, "How is your sex life"? This question was organized into a 7-point Likert scale ranging from 1 (good, as usual) to 7 (bad and inactive). It is important to note that higher scores for the items representing perception of marital satisfaction indicate increased marital dissatisfaction.

\section{Control variables}

Consistent with the marital quality and depression literature (Whisman, 2001), age, income, education, and race were included in the analysis as control variables. Age was mea- 
sured by the respondents' reported years. Income was based on minimum salary. Because of high past inflation, Brazil adopted a system for salaries that fluctuates based on changes in inflation. This metric is called a "minimum salary." So whereas the United States bases income on a specific dollar amount, Brazil bases it on the purchasing power of their currency. Education was measured in terms of years of schooling.

Although the entire sample is considered to be Latina by virtue of being in Brazil, race was used to factor in the potentially differing experiences of Black-Brazilian Latinas from White-Brazilian Latinas. The primary intent of the study was to understand the association between marital satisfaction and depression among Latinas, and all individuals participating in the study were born and raised in a Latino nation and are considered culturally Latina regardless of race. However, because of this racial diversity, it was important to include race in the analysis to assess within-culture differences. These racial categories were Black, White, Mixed race, and other.

\section{Analysis}

A structural equation modeling (SEM) method of analysis was used to analyze the data. The analysis program, AMOS (Arbuckle, 1999), which uses graphic models to represent the theoretical construct, computed model outputs. Figure 1 illustrates the model that was tested.

SEM is generally superior to standard multiple regression when analyzing survey data because it accounts for measurement error, which leads to more accurate estimates of the associations between variables (Kline, 1998). The first step of analysis was to verify the model's goodness of fit. Several fit statistics were used to indicate that the model accurately represented the data. After determining that the model fit the data, the second step was to test the hypotheses of the study. Path coefficients for latent variable associations were used to verify or refute the hypotheses. These path coefficients are reported in unstandardized and standardized coefficients, with the degree of significance reported as a $p$ value.

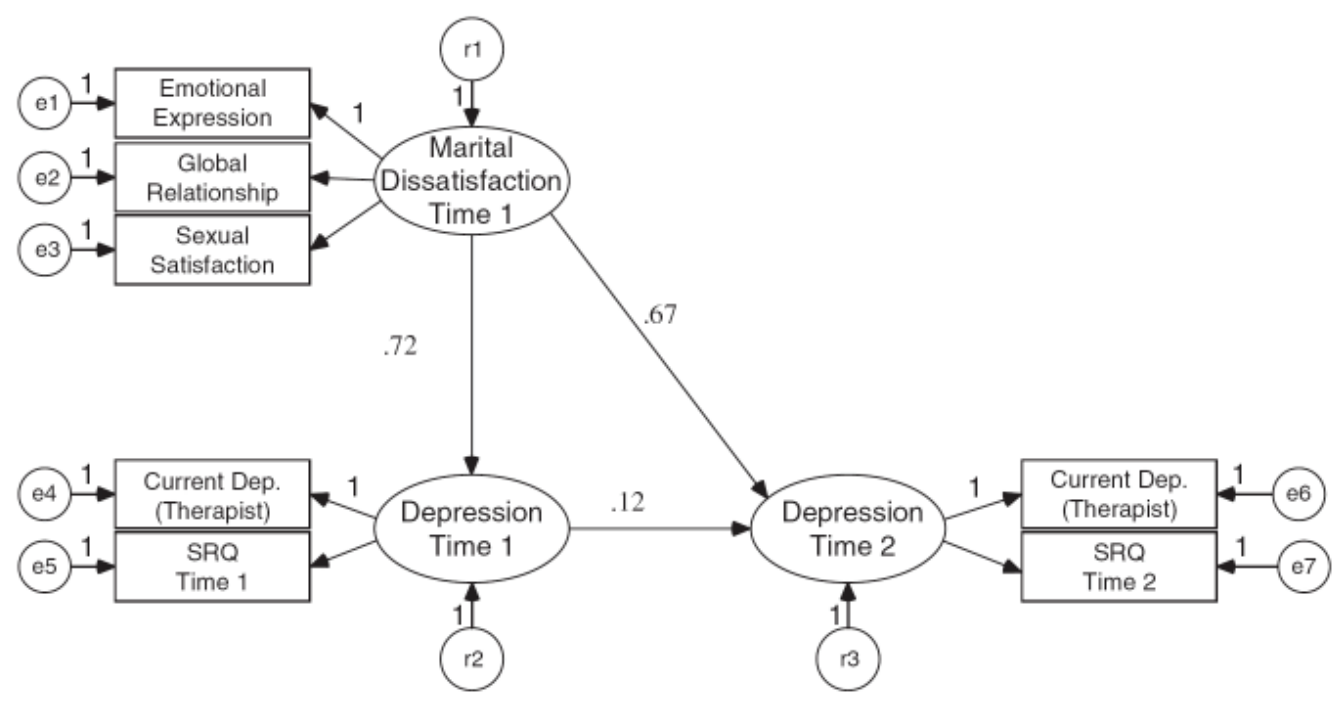

Figure 1. Full Hypothesized Conceptual Model with Standardized Path Coefficients 


\section{RESULTS}

\section{Descriptive Results}

Before reporting on the hypotheses tests, a brief examination of the descriptive statistics of key variables will contextualize the study. The SRQ-20 mean for Time 1 was 5.1 ( $S D=$ $3.73)$ and $5.4(S D=3.73)$ for Time 2. The scores had a range of 19 at Time 1 and 18 at Time 2. Scores on the therapist assessment of depression ranged from 1 to 3 and represent degree of depression severity, with 1 indicating no evidence of depressive disturbance and 3 indicating serious disturbance with depression. The mean for Time 1 was $2.08(S D=.94)$, and for Time 2 , it was $1.49(S D=.63)$. The mean for the global relationship item was 1.19 , with a range of 2 and standard deviation of .47. The mean for satisfaction with communication was 1.47 , with a range of 2 and a standard deviation of .72. The range for sexual satisfaction was 6 , with a mean of $2.67(S D=1.77)$. The mean for the summed three items of the marital satisfaction scale was 5.33, with scores ranging from 3 to $12(S D=2.34)$. In Table 1 , the correlation matrix lists associations between variables.

\section{Control Variables}

Results indicated that none of the control variables was statistically significant in the model. The standardized regression value for age was $.19(p=.11)$, race was $-.06(p=.60)$, income was $.13(p=.25)$, and education was $-.19(p=.10)$. These results suggest that the relationship between marital satisfaction and depression was independent of age, socioeconomic status (SES), education, and inter-Latina racial variation. Variables in the SEM model that are not theoretically necessary and for which there is no significant relationship should be omitted from the final model to improve parsimony (Byrne, 2001). Because the control variables were neither influential nor necessary for the functioning of the model, they were omitted from the final model in which the hypotheses were tested.

\section{Structural Model of Marital Dissatisfaction and Depression}

Before the full model was tested, it was necessary to test the model identification between Time 1 and Time 2 depression. Results indicated that the depression latent factors were significantly related at Time 1 and Time 2 . The model fit statistics were adequate. The standardized regression coefficient between the depression latent variables was .63 $(p<.000$, unstandard-

TABLE 1

Intercorrelations Between Marital Quality and Depression Items $(N=99)$

\begin{tabular}{lccccccc}
\hline Items & $\mathbf{1}$ & $\mathbf{2}$ & $\mathbf{3}$ & $\mathbf{4}$ & $\mathbf{5}$ & $\mathbf{6}$ & $\mathbf{7}$ \\
\hline 1. Communication satisfaction & - & $.40^{* *}$ & $.39^{* *}$ & $.36^{* *}$ & $.29^{* *}$ & $.38^{* *}$ & $.35^{* *}$ \\
2. Global relationship satisfaction & & - & $.39^{* *}$ & $.47^{* *}$ & $.27^{* *}$ & $.44^{* *}$ & $.38^{* *}$ \\
3. Sexual satisfaction & & & - & $.33^{* *}$ & $.23^{*}$ & $.31^{* *}$ & $.32^{* *}$ \\
4. Therapist Time 1 rating & & & & - & $.60^{* *}$ & $.39^{* *}$ & $.36^{* *}$ \\
5. SRQ Time 1 & & & & & - & $.37^{* *}$ & $.48^{* *}$ \\
6. Therapist Time 2 rating & & & & & & - & $.64^{* *}$ \\
7. SRQ Time 2 & & & & & & - \\
\hline
\end{tabular}

${ }^{*} p<.05 ; p^{* *}<.01$. 
TABLE 2

Regression Weights for the Full Model (Standard Errors in Parentheses; $N=99$ )

\begin{tabular}{lccl}
\hline Parameter Estimate & Unstandardized & Standardized & $\boldsymbol{p}$ \\
\hline Mar qual $\mathrm{t} 1 \rightarrow$ Depression $\mathrm{t} 1^{* * *}$ & $1.40(.35)$ & .72 & .000 \\
Depression $\mathrm{t} 1 \rightarrow$ Depression $\mathrm{t} 2$ & $.08(.15)$ & .12 & .60 \\
Mar qual $\mathrm{t} 1 \rightarrow$ Depression $\mathrm{t}{ }^{*}$ & $.83(.34)$ & .67 & .014 \\
\hline
\end{tabular}

${ }^{*} p<.05 .{ }^{* * *} p<.001$.

$X^{2}=14.09(p=.23, \mathrm{df}=11) ; \mathrm{GFI}=.93, \mathrm{TLI}=.97, \mathrm{RMSEA}=.05$.

ized regression $=.44)$. SEM analysis computes a squared multiple correlation coefficient that, like the $R^{2}$ in multiple regression, represents the percent of variable change explained by the exogenous variables influencing it. The squared multiple correlation indicated that depression at Time 1 predicted $40 \%$ of the variance for depression at Time 2 .

Analysis of the full model was then calculated to the test the study's hypotheses. The goodness-of-fit tests indicated that the model fit the data well. The chi-square for the full model was 14.09 ( $p=.23, d f=11, N=99)$, the GFI was .93, the TLI was .97, and the root mean square error of approximation (RMSEA) was .05. Figure 1 illustrates the results of the final model, using standardized path coefficients. The results are also reported in Table 2. These results indicate that there was a significant relationship between depression at Time 1 and marital satisfaction at Time 1 , with a standardized path coefficient of $.72(p<.001)$, which supports the first hypothesis. The second hypothesis was also supported, with Time 1 marital satisfaction being a significant predictor of Time 2 depression (standardized coefficient $=.67, p<.05$ ). In this model, Time 1 marital satisfaction was predictive of $52 \%$ of the variance for Time 1 depression. When controlling for Time 1 marital satisfaction, Time 1 depression was not a significant predictor of Time 2 depression (standardized coefficient $=.12, p=.60$ ). Time 1 depression and Time 1 marital satisfaction combined to explain $57.6 \%$ of the variance of Time 2 depression.

\section{DISCUSSION}

\section{Hypothesis 1}

For women in this Brazilian sample, the level of marital satisfaction at Time 1 predicted the depression at Time 1, independent of age, SES, and racial variation. This finding is consistent with the recent study in the United States that used a similar model to test the predictive ability of marital satisfaction on subsequent depression (Beach et al., 2003). In their model, the relationship between Time 1 marital satisfaction and Time 1 depression yielded a standardized parameter estimate of $-.49(p<.01)$. In this Latina model, the standardized parameter estimate for this relationship was $.72(p<.001)$. (It is important to note that, because of inverted marital satisfaction scoring, it appears that the relationship between variables between studies is inverted, whereas in reality, they are directionally synonymous.) Although the use of different measures prevents statistical comparisons between the two models, informal comparisons of the standardized parameter estimates suggest that marital satisfaction may have a stronger cross-sectional relationship with depression among Brazilian women than women in the United States. 


\section{Hypothesis 2}

For this Latina sample, marital satisfaction at Time 1 was predictive of depression at Time 2. The association was much stronger for this population than that reported by Beach et al. (2003), who reported a path coefficient of $-.15(p<.05)$. This was true even when controlling for age, SES, education, and race. This outcome may indicate cultural differences regarding the role that marital satisfaction plays in the Latina development of depression.

Of particular note is the lack of significance of the relationship between depression at Time 1 and Time 2, when controlling for Time 1 marital satisfaction scores. This finding differs from that of Beach et al. (2003), who found a more significant relationship between depression at Time 1 and Time 2 than they did between marital satisfaction at Time 1 and depression at Time 2. The difference may be due to the increased association between marital satisfaction and depression among the Latino population. Time 1 marital satisfaction may subsume much of the predictive power of Time 1 depression. This finding indicates the importance of marital distress as a predictor of subsequent depressive symptoms among the Latina population and suggests that marital satisfaction is a stronger predictor than depressive symptomotology of future depression.

The findings of this study provide evidence that the Marital Discord Model of Depression (Beach et al., 1990) is applicable to Latina populations, which suggests that marital discord both causes and maintains depressive symptomatology. These findings are significant because it is the first study to relate marital satisfaction longitudinally to depression among Latinas. It also suggests that the association between variables may be greater among Latinas than among Whites.

Although Beach's model describes marital discord as an important antecedent of depression, it should be noted that there is evidence that depressive symptoms can also be a predictor of marital satisfaction. With the heterogeneity of depression etiology, it is not surprising that there is empirical evidence that supports both scenarios (Beach \& O'Leary, 1993; Prince \& Jacobson, 1995). In situations in which depression predates marriage, it is logical that depression leads to subsequent marital distress. Other times, marital distress is a clear precursor to subsequent depressive symptoms. After reviewing the research literature, Prince and Jacobson concluded that marital distress can be "an antecedent, concomitant, and consequence of depression" (p. 407). Even Beach recognized the reciprocal nature of the etiology of marital distress and depression (Beach et al., 2003). Nevertheless, there is substantial evidence that marital distress leads to subsequent depression, and the treatment of marital distress alleviates depressive symptomatology (Beach, 2001; Dessaulles, Johnson, \& Denton, 2003; Prince \& Jacobson), which makes the validation of Beach's model across different cultures important.

\section{Limitations and Directions for Research}

Because culture has been hypothesized as a moderating variable between marital satisfaction and depression (Whisman, 2001), the present study was more aptly poised to explicitly test its influence on the relationship between these variables because it was conducted within the native Latino culture. This characteristic helped to isolate the impact that marital satisfaction had on the etiology of depression among Latinos, while eliminating factors related to 
the process of acculturation that one finds in studies done with Latinos in the United States. Thus, by conducting the study in a Latino country, no measure of acculturation was needed. In the cross-sectional American Latino study relating marital satisfaction to depression (Vega, Kolody, \& Valle, 1988), acculturation was found to be a moderating factor between the variables. The present study was conducted with Latinos in their native country, thereby eliminating the influence of acculturation on the relationship between marital satisfaction and depression. However, it is recognized that further research is needed among Latino Americans, in which indicators should be included to test the influence of acculturation.

Just as there is variance between cultures, there is also variance within cultures. Because there is great diversity between Latino cultures, it is important not to generalize these findings to all Latino populations. Further research needs to be conducted to test if these results are consistent among other native Latino cultures (e.g., Mexico, Puerto Rico, Cuba, and so on) and variability among Latinos living in the United States (e.g., Mexican Americans, Puerto Rican Americans, Brazilian Americans, Cuban Americans, and so on). Consequently, additional research needs to be done to effectively generalize these findings to the broader Latino population.

In addition, assessment instruments need to be developed and validated for construct evaluation (i.e., marital satisfaction). This lack of non-English standardized assessment instruments makes it difficult to explicitly compare these results with the Beach et al. (2003) study. However, this is not the first longitudinal marital satisfaction and depression study to use psychometrically untested measures of marital satisfaction. For example, Whisman and Bruce (1999) used a single question to assess marital satisfaction. However, future research with Latino populations that uses standardized instruments will improve the internal and external validity of the study. Similarly, further research needs to assess marital satisfaction, as well as depression, longitudinally to evaluate the bidirectionality of the relationships between the variables.

Another limitation is that only data from the mothers were used in this study. Whisman (2001) noted that several studies have identified different degrees of association between marital quality and depression for men and women. Because the present study did not collect data from the fathers at Time 2, the ability to describe the interrelationship between husband and wife marital satisfaction was eliminated.

Beach et al. (2003) noted that gender differences may be due to stereotypical perceptions of responsibility for the marital relationship. Family structures influence the degree of perceived responsibility for marital quality. Because Latino couple relationships have been characterized as hierarchical, with women assuming a greater responsibility for maintaining the relationship (Korin, 1996), it stands to reason that gender differences related to the associations between marital satisfaction and depression may be more pronounced among Latinos. Further research needs to be done to assess gender differences and the cross-spouse influence on depression among Latinos.

\section{Clinical Implications}

The preliminary validation of the Marital Discord Model of Depression with a Latina population suggests the utility of using couples therapy to treat depression among Latinas in cases in which part of the depressive symptomatology is related to marital distress. A number of models of couples therapy treat depression within a relational context, including cognitive 
marital therapy, strategic couple therapy, and conjoint interpersonal therapy, behavioral marital therapy for depression (BMT-D) and emotionally focused therapy (EFT; Gollman, Friedman, \& Miller, 2002). The latter two models have been empirically validated for the relational treatment of depression.

BMT-D is based on several assumptions regarding the way marital satisfaction affects depression. The developers of this model proposed that there are six aspects of marital interaction that act as a source of defense against depression, and five aspects of the marital relationship that have the potential to exacerbate depressive tendencies (Beach et al., 1990). Interventions are designed to strengthen the couple's ability to use the six marital defense mechanisms. However, there is no empirical evidence to indicate whether these marital defense mechanisms and risk factors are culturally applicable to the Latino population.

Research has validated the use of BMT for the treatment of depression (Beach, 2001; Prince \& Jacobson, 1995). BMT-D has been shown to be effective at treating both marital discord and concurrent depression (Beach). Effectiveness seems to be greatest when the couple with a depressed person reports marital dissatisfaction as a factor in the depressed episode. Treating depression and marital satisfaction through BMT-D has been shown to be more effective for alleviation of depression symptoms than the cognitive therapy treatment of depression alone when marital dissatisfaction is coexisting (Addis \& Jacobson, 1996).

EFT also includes marital dissatisfaction in the conceptualization of depression, as well as its resolution, using the treatment of marital interactions as the intervention strategy for depression (Johnson, 1996). An outcome study found that EFT was equally effective at reducing depression symptomatology, as was pharmacotherapy (Dessaulles et al., 2003). Although no significant pre- to post-treatment differences existed between EFT and pharmacotherapy, there was a difference at the 6-month follow-up. The depressive symptomatology for the couples who participated in EFT continued to decrease over the 6-month follow-up, whereas those in the pharmacotherapy group reported no differences from post-treatment to 6-month follow-up

Although BMT-D and EFT have both been shown to be effective in treating depression from the perspective of addressing marital discord, none of the therapy models, including cognitive marital therapy, strategic couple therapy, and conjoint interpersonal therapy, has been tested with Latinos. Sprenkle (2002) noted that a common weakness of clinical outcome research is the lack of cultural diversity; such is the case with the relational treatment of depression. Further research is needed to test the effectiveness of couples therapy in treating depression among Latinos. This point is made more urgent by the results from this study that provide support for the Marital Discord Model of Depression in a Latina population.

\section{REFERENCES}

Addis, M.E., \& Jacobson, N.S. (1996). Reasons for depression and the process and outcome of cognitive-behavioral psychotherapies. Journal of Consulting and Clinical Psychology, 64, 1417-1424.

Almeida-Filho, N., Mari, J.J., Coutinho, E., Andreoli, S., Fernandes, J., \& Franca, J. (2002). Brazilian multicentric study of psychiatric morbidity: Methodological features and prevalence estimates. Brazilian Journal of Psychiatry, 171, 524-529. 
American Psychiatric Association. (2000). Diagnostic and statistical manual of mental disorders (4th ed. text revision). Washington, DC: Author.

Arbuckle, J.L. (1999). Amos (Version 4.1) [Computer software] Chicago: SmallWaters.

Beach, S.R.H. (2001). Marital therapy for co-occurring marital discord and depression. In S.R.H. Beach (Ed.), Marital and family process in depression: A scientific foundation for clinical practice (pp. 205-224). Washington, DC: American Psychological Association.

Beach, S.R.H., Katz, J., Kim, S., \& Brody, G.H. (2003). Prospective effects of marital satisfaction on depressive symptoms in established marriages: A dyadic model. Journal of Social and Personal Relationships, 20, 355-371.

Beach, S.R.H., \& O'Leary, K.D. (1993). Marital discord and dysphoria: For whom does the marital relationship predict depressive symptomatology? Journal of Social and Personal Relationships, 10, 405-410.

Beach, S.R.H., Sandeen, E.E., \& O'Leary, K.D. (1990). Depression in marriage. New York: Guildford Press.

Beck, A.T., Rush, A.J., Shaw, B.F., \& Emery, G. (1982). Terapia cognitive da depressão [Cognitive therapy of depression] (V. Ribeiro, Trans.). Rio de Janeiro, Brazil: Zahar.

Byrne, B.M. (2001). Structural equation modeling with AMOS: Basic concepts, application, and programming. Mahwah, NJ: Erlbaum.

Dehle, C., \& Weiss, R.L. (1998). Sex difference in prospective associations between marital quality and depressed mood. Journal of Marriage and the Family, 60, 1002-1011.

Dessaulles, A., Johnson, S.M., \& Denton, W.H. (2003). Emotion-focused therapy for couples in the treatment of depression: A pilot study. American Journal of Family Therapy, 31, 345-353.

Gollman, J.K., Friedman, M.A., \& Miller, I.W. (2002). In A.S. Gurman \& N.S. Jacobson (Eds.), Clinical handbook of couple therapy (3rd ed, pp. 653-676). New York: Guilford Press.

Gorenstein, C., Andrade, L., Filho, A.H.G.V., Tung, T.C., \& Artes, R. (1999). Psychometric properties of the Portuguese version of the Beck Depression Inventory on Brazilian college students. Journal of Clinical Psychology, 55, 553-562.

Gottman, J.M. (1999). The marriage clinic: A scientifically based marital therapy. New York: W. W. Norton.

Harding, T.W., De Arango, M.V., Baltazar, J., Climent, C.E., Ibrahim, H.H.A., \& Ladrido-Ignacio, L., et al. (1980). Mental disorders in primary health care: A study of their frequency and diagnoses in four developing countries. Psychological Medicine, 10, 231-241.

Johnson, S.M. (1996). The practice of emotionally focused marital therapy: Creating connection. New York: Brunner/Mazel.

Kline, R.B. (1998). Principles and practices of structural equation modeling. New York: Guilford Press.

Korin, E.C. (1996). Brazilian families. In M. McGoldrick, J. Giordano, \& J.K. Pearce (Eds.), Ethnicity and family therapy (2nd ed, pp. 200-213). New York: Guilford Press.

Mari, J.S., \& William, P.A. (1986). A validity study of psychiatric screening questionnaire (SRQ-20). British Journal of Psychiatry, 42 (Suppl. 1): 55-60.

Miller, R.B., \& Wright, D.W. (1995). Detecting and correcting attrition bias in longitudinal family research. Journal of Marriage and the Family, 57, 921-929. 
Parada, B.V., Stevenson, P.R., \& Saldivia, B.S., Lohn, R., \& Torres, S.P. (2002). Estudio Chileno de prevalencia de patologia psiquiatrica: DSM-III-R/CIDI, ECPP [Chilean study of prevalence of psychiatric disorders]. Review of Medicine in Chile, 130, 527-536.

Prince, S.E., \& Jacobson, N.S. (1995). Couple and family therapy for depression. In E.E. Beckham \& W.R. Leber (Eds.), Handbook of depression (2nd ed, pp. 404-424). New York: Guilford Press.

Schafer, R.B., Wickrama, K.A.S., \& Keith, P.M. (1998). Stress in marital interaction and change in depression: A longitudinal analysis. Journal of Family Issues, 19, 578-594.

Sprenkle, D.H. (2002). Effectiveness research in marriage and family therapy. Alexandria, VA: American Association for Marriage and Family Therapy.

Vega, W.A., Kolody, B., \& Valle, R. (1988). Marital strain, coping and depression among MexicanAmerican women. Journal of Marriage and the Family, 50, 391-403.

Whisman, M.A. (2001). The association between depression and marital dissatisfaction. In S.R.H. Beach (Ed.), Marital and family processes in depression: A scientific foundation for clinical practice (pp. 3-24). Washington, DC: American Psychological Association.

Whisman, M.A., \& Bruce, M.L. (1999). Marital dissatisfaction and incidence of major depressive episode in community sample. Journal of Abnormal Psychology, 108, 674-678. 